\title{
OPTICAL OBSERVATIONS IN ESA'S SSA PROGRAMME
}

\author{
B. Jilete ${ }^{1}$, A. Mancas ${ }^{2}$, T. Flohrer ${ }^{3}$, and H. Krag ${ }^{4}$
}

\begin{abstract}
\end{abstract}
has been undertaking a Space Situational Awareness (SSA) Programme with three segments: Space Weather (SWE), Near Earth Objects (NEO) and Space Surveillance and Tracking (SST).

The main objective of SSA, as an optional ESA programme, is to enable Europe to autonomously detect, predict and assess the risk to life and property due to man-made space debris objects, re-entries, in-orbit explosions, in-orbit collisions, disruption of missions and satellite-based service capabilities, potential impacts of Near Earth Objects (NEOs), and the effects of space weather phenomena on space and ground-based infrastructure.

The ESA SSA SWE Segment is in charge of monitoring the environmental conditions in Earth's Magnetosphere, Ionosphere and Thermosphere due to the Sun and solar winds that can influence the functioning and reliability of space and on-ground assets, as well as human health. For the operational systems measurement systems and services are re- 
quired. This requires a constant monitoring of the space environment and timely dissemination of nearreal-time information, warnings and forecast of upcoming space weather events to users in industry, government and research institutes.

The vast European expertise and already established assets of observations, results, models and products are used in federated space-weather serviceprovision concept. These space weather services are centralised at ESA's Space Weather Coordination Centre (SSCC) located at the Space Pole in Belgium providing Europe's first Space Weather Helpdesk. Further, the segment is connecting a federated network of Expert Service Centres (ESCs) across Europe, the SWE Data Centre at ESA's Redu Centre, and various discipline-specific Data Centres within SSA Member States.

The ESA SSA NEO Segment is in charge of cataloguing and predicting the trajectories of Near Earth Objects, i.e. comets and asteroids with sizes ranging from meters to tens of $\mathrm{km}$ that orbit the Sun and whose orbit can come close to the Earth. The segment is expanding the knowledge of the current and future position of NEOs relative to Earth by actively observing NEOs and coordinating Europe's role in the global asteroid hunt through syndicating and federating Europe's current observation capabilities and data sources. New sky-survey technologies and efficient data and information handling networks are developed. Further, the segment predicts orbits, produces impact warnings by estimating the likelihood of Earth impacts and assessing the consequences of any possible impact, and develops NEO deflection methods and mitigation measures.

The ESA SSA SST Segment is in charge of detecting and predicting the movement of man-made space objects in space. This allows applications, such as to determine potential collision between objects, to predict upcoming re-entries and to detect fragmentations (by explosion or collision). In SST, the development of the technologies for detection, cataloguing and follow-up of space objects, and of derived applications for conjunction events prediction, re-entry predictions, and fragmentation events predictions are considered as the first important steps towards a European SST capability. To achieve this goal, ESA is:

- Focusing on research and development, supporting national initiatives.

- Developing cross-national SST components and technologies (sensors and data processing).

- Supporting the deployment of expert centres to pursue the qualification of national assets and net- working technologies.

- Ensuring interoperability through common processing, formats, architecture analysis.

Following sections will describe as examples three different activities carried out under ESA SSA Programme SST Segment that represented a subset of the activities related to optical observations: optical passive measurements qualification analysis, SST Expert Centre and Test-bed robotic telescopes.

\section{QUALIFICATION DATA ANALYSIS: SHOT TELESCOPE}

Under the frame of a Support observations and sensor qualification campaign, several sensors were analysed and proposed. SHOT telescope of Teplice Observatory in Czech Republic, which belongs to North Bohemian Observatory and Planetarium network, participated in the campaign. This telescope is a $0.43 \mathrm{~m} \mathrm{f} / 6.8$ corrected Dall-Kirkham telescope with a precise German equatorial mount (10micron GM3000 HPS) and an Apogee Aspen CG9000 $(3056 \times 3056)$ CCD camera. It was qualified for the first time in the SST context (satellites and active debris) observations in mid-2017.

The data that was analysed came from a dedicated observation campaign, which objectives were the acquisition of observations to further use in endto-end Software testing and validation, and the support to test and validate development activities, performing survey and tracking experiments with optical passive as well as laser ranging sensors.

The qualification analysis covered observations of two satellites: NAVSTAR 46 GPS satellite and Sentinel-2A Earth Observation Satellite. Sentinel2B observations were used to verify measurement biases. These observations were delivered by SHOT to ESA in CCSDS TDM format [CCSDS (2007)]. SHOT provided astrometric information (right ascension and declination) and photometric data (apparent magnitude). The reference orbits to compare with aforementioned satellites were obtained from different sources, IGS FTP servers and CODE for NAVSTAR 46, and ESA Navigation Support Office for both Sentinel satellites. The software used for the analysis was ESA'S NAPEOS libraries [NAPEOS (2013)]. The steps followed for the performance estimation were:

1. Application of the annual aberration to the data delivered by SHOT.

2. Determination of the time bias using reference orbits using all GPS satellites for NAVSTAR 46 and all Sentinel satellites for Sentinel-2A and corrected orbits obtained from step 1. 


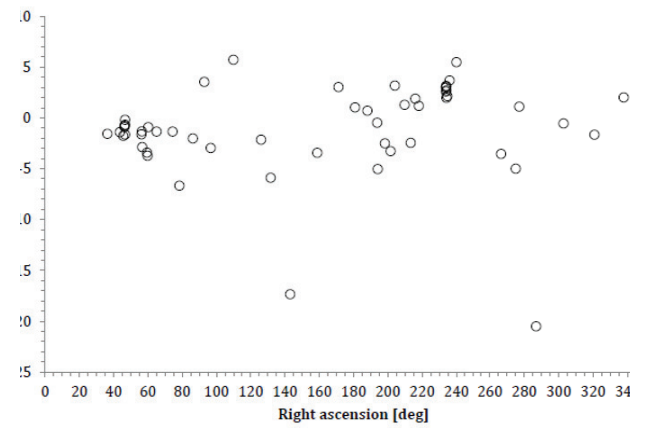

Fig. 1. Sentinel-2A right ascension residual (multiplied by the cosine of declination) against right ascension after bias correction.

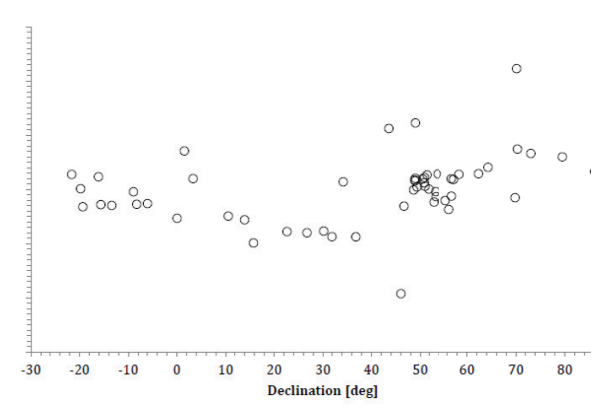

Fig. 2. Sentinel-2A declination residuals against declination after bias correction.

3. Computation with NAPEOS the observation residuals relative to the reference orbits.

4. Perform batch OD including one-week forward propagation.

5. State vector (position and velocity) residuals computation between OD orbit and reference orbit.

The overall results of this first analysis were quite promising: epoch bias in the order of 55 $\mathrm{ms}$, and RADEC biases within the accuracy envelope for both MEO and LEO orbits defined in the SST System Requirement Document [ESA-SSAteam (2013)]. SENTINEL-2A analysis reveals a bias between -5 to -15 arcsec in Right Ascension, between -5 to -10 arcsec in Declination and an epoch bias around $55 \mathrm{~ms}$. Figure 1 and Figure 2 gathered the residuals re-computation after taking into account those biases.

NAVSTAR-46 analysis reveals a small bias around -0.4 arcsec in Right Ascension (Figure 3), around -0.85 arcsec in Declination (Figure 4) and an epoch bias around $55 \mathrm{~ms}$.

Overall, the results prove that the sensor can be used for SST observations and the resulting orbits meet the SST System Requirement Document accuracy requirements.

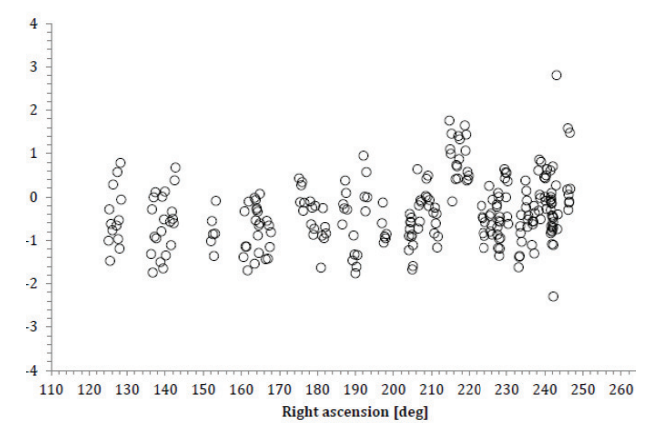

Fig. 3. NAVSTAR-46 right ascension residual (multiplied by the cosine of declination) against right ascension.

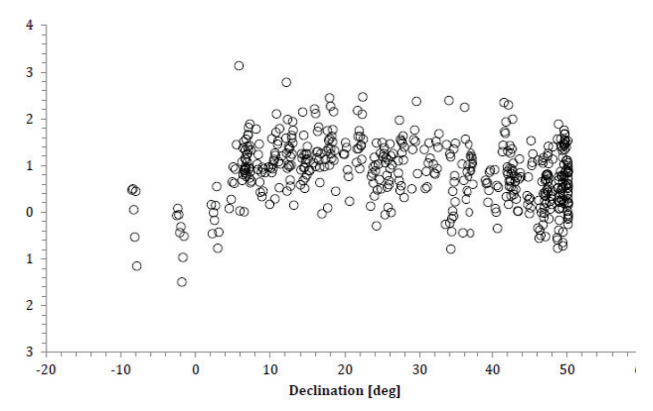

Fig. 4. NAVSTAR-46 declination residuals against declination.

\section{SST EXPERT CENTRE}

In SST a certain overhead in coordinating observations requests can be expected from an architecture that is incorporating to a larger extend existing sensors under external control. This is mainly due to very practical aspects, such as adopted data formats and general interfaces, and due to the usually very different sensor capabilities and availability schemes. Therefore, the main task of the Expert Centre is to be a proxy between the SST segment and the sensors and to provide a single transparent interface for data acquisition, support services, research and technology development. In any case the architecture foresees that the Expert Centre is always external to the SST system and will not replace any core SST functionality.

[Jilete et al. (2015)] presented the outcome of an effort to analyse the benefits of a SST expert centre, the core set of requirements and draft system architecture. The following benefits of establishing a SST Expert Centre as a proxy between two sides (SST Backend and external sensors) were identified [Flohrer et al. (2015)]:

- Lower costs, functionality increases, performance gains. 


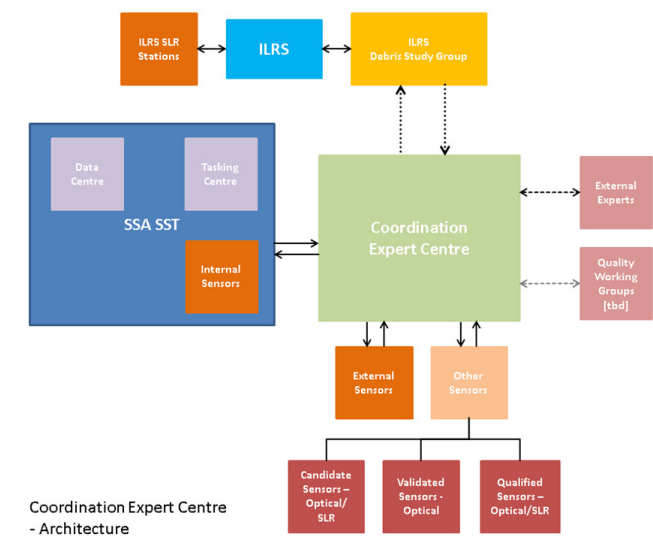

Fig. 5. Expert Centre system architecture.

- Expert support to the external sensors.

- Data quality checks and applying consistent modelling.

- Centralized scheduler meeting backend SST/sensor status.

- Standardisation of data exchanges.

- Test environment for advanced SST data processing techniques.

The aforementioned benefits show that the Expert Centre will have two-fold top level functions: operational and support functions. The operational functions are:

- Coordinate the sensors for tracking and surveillance tasks.

- Qualify external data sources.

- SLAs monitoring.

The support functions are:

- Calibration of sensors.

- Evaluation of observation and data processing techniques.

- Data quality control.

- Expert support (considering both types of experts, internal and external to the Expert Centre, e.g. ILRS Debris Study Group).

- Research and development.

In 2016 the development activities have been started and the requirements have been re-visited to arrive at a matching system design [Flohrer et al. (2016)]. Figure 5 depicts the high level interfaces between the Expert Centre, the backend SST system, and the internal and external (federated) optical and SLR sensors. It is important to bear in mind that this Expert Centre concept will be an external component to a SST system, and none of the central SST functionalities will be replaced, which of course calls for the difficult task of an efficient mitigation of duplication of observation requests.
In 2017 a first combined Expert Centre optical passive and laser ranging measurements prototype has been installed and deployed at ESA. The development activities are expected to continue in the period 3 of the SSA Programme (2017-2019) with the finalisation of the implementation, deployment, and extensive test campaigns with both optical and SLR sensors. In this testing all Expert Centre functionalities and performance will be validated.

\section{TEST-BED ROBOTIC TELESCOPE}

The TBT project, "Demonstration Test-Bed for the Remote Control of an Automated Follow-Up Telescope" is being developed under ESA's General Studies and Technology Programme (GSTP), with the objective of implementing a test-bed for the validation of an autonomous optical observing system. The system consists of two fully robotic telescopes, one in ESA's ESTRACK Cebreros Satellite Tracking Station, Spain and the other in La Silla, Chile. The former is currently under commissioning phase and the later it is foreseen its installation during 2018. The telescope is a fast astograph with a large Field of View of $2.5 \times 2.5$ square-degrees and a plate scale of 2.2 arcsec per pixel. The tube is mounted on a fast direct-drive mount moving with speed up to 20 degrees per second. The focal plane hosts a 2-port $4 \mathrm{~K} \times 4 \mathrm{~K}$ back-illuminated $\mathrm{CCD}$ with readout speeds up to $1 \mathrm{MHz}$ per port. All these characteristics ensure good survey performance for transients and fast moving objects.

Detection software and hardware (based on COTS) were optimised for the detection of NEOs and objects in high Earth orbits. Nominal exposures are in the range from 2 to 30 seconds, depending on the observational strategy. Part of the validation scenario involves the scheduling concept integrated in the robotic operations for both sensors. Every night it takes all the input needed and prepares a schedule following predefined rules allocating tasks for the telescopes. Telescopes are managed by RTS2 control software [8], that performs the real-time scheduling of the observation and manage the ancillary hardware. At the end of the observations, the observing systems report astrometric positions and photometry of the objects detected.

There is a control layer executing the tasks defined by the scheduler, between the functional layer and the monitoring front-end of the HMI. Figure 6 depicts the control module top-level architecture.

The scheduling tool implements two top-level observing strategies, survey and follow-up, for both NEOs and high-orbit satellites. The survey strategy aims at the detection of new objects for different 


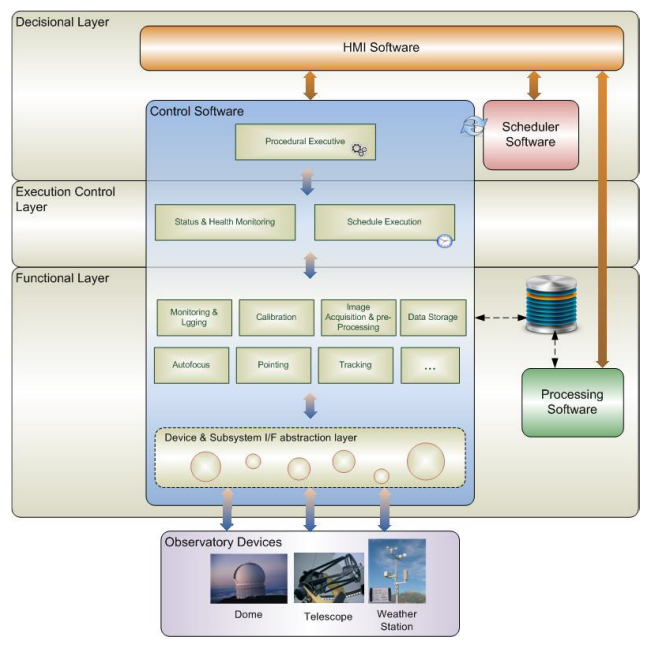

Fig. 6. TBT control module top-level architecture [Ocaña et al. (2016)].

target population, whereas the follow-up goal is the detection of objects in need of revisit. The scheduling tool is a web-based planning tool for the manual and automatic planning. It generates the observation plan and relies on the RTS2 internal meta-queue system for the real-time response and monitoring of the system.

The control software, RTS2 [Kubánek (2010)], is an open source package with the aim to create a modular environment for complete observatory control. It consists of several independent programs. Those programs communicate via RTS2 protocol, acting as client and as server, based on TCP/IP connections.

The data processing is done on-site of each sensor. A first basic post-processing is done by RTS2, correcting images from bias and flats. The selected based on COTS software was TOTAS [Koschny (2015)]. This software was adapted to include satellite images (MEO and GEO satellites) processing capabilities, based on masking the trailed stars in images taken with on-object tracking.

The telescope located in Cebreros is already working in robotic but manual mode and under commissioning phase. Some validation and qualification observation campaign were conducted within the Expert Centre activity scope. First analysis, conducted by using the first deployed version of the Expert Cen- tre, shows an astrometric accuracy in the order of 1.6 arcsec, and further improvements are foreseen from other ongoing projects.

\section{SUMMARY}

The increasing space traffic requires acquiring accurate and timely observations of space objects. Therefore, the objectives behind the observations campaigns in SST are not only data acquisition but also qualification and coordination of sensors.

The expert centres will establish a robust and flexible solution to coordinate in an optimise way external sensors, and to provide expertise in calibration and qualification of sensors for SST context.

Progress in ESA's test-bed robotic telescopes project was also briefly described. The first telescope installed in Cebreros is already working after conducting the commissioning phase, in robotic mode. First analysis shows an astrometric accuracy of 1.6 arcsec, and further improvements are foreseen from other ongoing projects.

\section{REFERENCES}

Flohrer, T., Jilete, B., Krag, H., Funke, Q., Braun, V. et al. 2016, Proc. 2016 ILRS Workshop, Potsdam

Flohrer, T., Jilete, B., Mancas, A., \& Krag, H., 2015, Advanced Maui Optical and Space Surveillance Technologies Conference, Maui, 2015, Hawaii

Jilete, B., Mancas, A., Flohrer, T., \& Krag, H. 2015, Proceedings of the 2015 ILRS Technical Workshop "Network Performance and Future Expectations for ILRS Support of GNSS, Time Transfer and Space Debris Tracking", Matera, Italy

Koschny, D. \& Busch, M. 2015, P\&SS, 118, 305

Kubánek, P. 2010, AdAst, 88

NAPEOS: http://www.esa.int/Our_Activities/ Operations/NAPEOS

Ocaña, F., Ibarra, A., Racero, E., Montero, A., Doubek, J. et al. SPIE, 9906, 66

SSA team. 2013, Space Situational Awareness - Space Surveillance and Tracking System Requirements Document: SSA-SST-RS-RD-0001_i1r5, October 2013

The Consultative Committee for Space Data Systems. 2007, Tracking Data Message, Recommended Standard CCSDS 503.0-B-1, November 2007 